УДК 547.022.1

\title{
Optimization of the Process of Synthesis of Lignin - Phenol -Formaldehyde Organic Aerogels
}

\author{
Nadezhda M. Mikova ${ }^{a}$, \\ Ludmila I. Grishechko ${ }^{\mathrm{a}}$ and Robert Z. Pen ${ }^{\mathrm{b} *}$ \\ ${ }^{a}$ Institute of Chemistry and Chemical Technology SB RAS \\ 50-24 Akademgorodok, Krasnoyarsk, 660036, Russia \\ ${ }^{b}$ Siberian State Technological University \\ 82 Mira, Krasnoyarsk, 660049, Russia
}

Received 24.03.2015, received in revised form 15.04.2015, accepted 15.05.2015

The method of experimental statistical analysis was applied to study the effect of mass ratio of phenol and lignin (factor $X_{1}$ ) and the weight ratio of thereof mixture with formaldehyde (factor $\left.X_{2}\right)$ on the values of specific parameters of the porous structure obtained organic lignin - phenolformaldehyde aerogels. Based on the evaluation of the mathematical model was established that under the observance terms of $X_{1}=0,65-0,78$ and $X_{2}=1,7$ the maximum predicted values of the specific surface area and mesopore volume of obtained organic aerogels are $485 \mathrm{~m}^{2} / \mathrm{g}$ and 1,83 $\mathrm{cm}^{3} / \mathrm{g}$, accordingly. Under optimal values of $X_{1}=0.25$, and $X_{2}=1.25$ calculated values reach a macropore volume of $4.05 \mathrm{~cm}^{3} / g$ and total pore volume $-4.67 \mathrm{~cm}^{3} / \mathrm{g}$.

Keywords: lignin, formaldehyde, lignin - phenol-formaldehyde gel, optimization process, mathematical model, porous structure, surface area, pore volume.

DOI: $10.17516 / 1998-2836-2015-8-2-287-300$.

(C) Siberian Federal University. All rights reserved

* Corresponding author E-mail address: inm@icct.ru 


\title{
Оптимизация процесса синтеза \\ лигнин-фенол-формальдегидных \\ органических аэрогелей
}

\author{
Н.М. Микова ${ }^{\mathrm{a}}$, Л.И. Гришечко ${ }^{\mathrm{a}}$, Р.3. Пен ${ }^{\mathbf{6}}$ \\ ${ }^{a}$ Институт химии и химической технологии СО РАН \\ Россия, 660036, Красноярск, Академгородок, 50/24 \\ ${ }^{6}$ Сибирский государственный технологический университет \\ Россия, 660049, Красноярск, пр. Мира, 82
}

Методом экспериментально-статистического анализа изучено влияние массового соотношения фенола и лигнина $\left(X_{1}\right)$ и массового соотношения их смеси с формальдегидом $\left(X_{2}\right)$ на значения параметров пористой структуры получаемых органических лигнинфенол-формальдегидный аэрогелей. На основании оценки полученной математической модели установлено, что при соблюдении условий $X_{1}=0,65-0,78$, а $X_{2}=1,7$ прогнозируемые максимальные значения удельной поверхности и объема мезопор получаемого органического

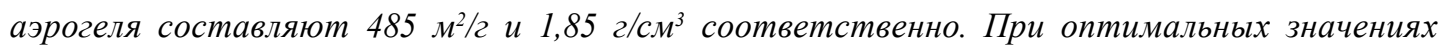
$X_{1}=0,25$, а $X_{2}=1,25$ рассчитанные значения объема макропор достигают 4,05 $\mathrm{cm}^{3} / 2$, а общего объема пор - 4,67 $\mathrm{cm}^{3} / 2$.

Ключевые слова: лигнин, формальдегид, лигнин-фенол-формальдегидный гель, прочесс оптимизации, математическая модель, пористая структура, удельная поверхность, объем nop.

\section{Введение}

Аэрогели представляют собой специфический класс материалов органической или углеродной природы с низкой плотностью и с большим объемом внутренних открытых пор, образованных полимерной структурой жестко связанных макрочастиц. Этот жесткий каркас занимает малую часть объема аэрогеля - почти весь объем приходится на поры [1]. Аэрогели как пористые материалы, обладающие низкой плотностью, высокой удельной поверхностью, низкой теплопроводностью, имеют широкие возможности для их дальнейшего применения. В органической форме гели могут быть использованы для доставки лекарственных средств [2], теплоизоляции [3], адсорбции летучих соединений, звукоизоляции и в катализе [4, 5]. Область применения аэрогелей варьируется в зависимости от их природы и конечных текстурных свойств, которые определяются условиями их приготовления.

Как правило, органические гели получают путем золь-гелевой полимеризации мономеров с альдегидами. Формальдегид является наиболее часто используемым альдегидом в качестве сшивающего агента при приготовлении гелей. Первый органический аэрогель, полученный из резорцина и формальдегида, был описан Пекала еще в 1989 г. К настоящему времени резорцинформальдегидные гели наиболее изучены и широко исследованы в различных областях [6-8]. 
Тем не менее высокая стоимость исходного сырья и многоступенчатая методика получения препятствует массовому производству резорцин-формальдегидные гелей, что мотивирует поиск новых путей синтеза и новых, более дешевых сырьевых источников, из которых можно получать подобные материалы.

Актуально использование доступных природных ресурсов в качестве сырья для получения гелей. Первые синтезированные органические гели из натуральных компонентов - это гели на основе целлюлозы [9-11]. Также сообщается о перспективном использовании хитина [12] и различных полисахаридов $[2,13]$ для получения аэрогелей. Известно, что и другие соединения, такие как фенол, полиолефины и танин, могут успешно заменить резорцин при получении органических гелей.

Авторами работ $[1,14,15]$ продемонстрировано, что аэрогели на основе фенола - это преимущественно микро-, мезопористые объекты, характеризующиеся хорошо развитой поверхностью до 600 г/м² и достаточно узким распределением пор по размеру в диапазоне от 2 до 4 нм. Пористость таких материалов может достигать $90 \%$.

Кроме того, танины могут успешно заменить резорцин при получении аэрогелей $[16,17]$. Наиболее высокая концентрация таких соединений сосредоточена в коре дерева, например сосны, дуба или мимозы.

Лигнин, имеющий в своем составе структуры фенольного типа, позволяет рассматривать его также в качестве перспективного сырьевого источника для получения пористых материалов с уникальными свойствами, в частности органических лигнин-фенол формальдегидных аэрогелей [18].

Проведенный анализ литературных источников показал, что актуальные направления исследований связаны с подбором доступных и экологически безопасных возобновляемых источников природного органического сырья для получения аэрогелей. Наиболее изучены такие параметры гелей, как кажущаяся плотность, общая пористость, удельный объем пор, распределение пор по размерам, удельная площадь поверхности и объем микро-, мезо- и макропор.

Аэрогель - объект с хрупкой структурой, которая обычно выращивается в жидкой фазе, а затем требуется освобождение жидкости, находящейся внутри него. Поэтому аэрогель можно получить, выполняя определенную цепочку технологических операций и поддерживая при этом требуемые параметры соответствующего процесса.

Задачей математической оптимизации синтеза органических лигнин-фенолформальдегидных аэрогелей являлась оценка влияния независимых факторов (концентрация исходных реагентов - лигнина и фенола, прореагировавших с формальдегидом) на значения выходных параметров пористой структуры получаемых из лигнина гелей (удельная поверхность, общий объем пор, объем мезопор и объем макропор).

\section{Экспериментальная часть}

Настоящая методика оптимизации осуществлена для математического моделирования условий, обеспечивающих наилучшие характеристики пористости при получении органических аэрогелей методом золь-гель-полимеризации лигнина и фенола, используемого в качестве сополимера, с формальдегидом. 
Математическая обработка результатов выполнена средствами пакета прикладных программ Statgraphics Centurion XVI, блок DOE (Design of Experiment), процедура Response Surface [19].

Было исследовано влияние двух переменных факторов:

$\mathrm{X}_{1}$ - массовое соотношение фенола и лигнина (в тексте и на рисунках принято обозначение «Фен/Л»);

$\mathrm{X}_{2}$ - массовое соотношение смеси лигнина с фенолом и формальдегида (в тексте и на рисунках «(Л + Фен) $/ \Phi »)$.

Остальные условия эксперимента: температура смешивания реагентов, температура и продолжительность гелеобразования (5 сут при $\left.85{ }^{\circ} \mathrm{C}\right)$, способ сушки, доля суммарного количества веществ в гелях в пересчете на сухую массу (лигнина, фенола, формальдегида) $26 \%$ оставались неизменными во всех опытах.

Результаты опытов характеризовали следующими выходными параметрами - свойствами аэрогелей:

$\mathrm{S}_{\text {Бэт }}$ - удельная поверхность, $\mathrm{M}^{2} / \Gamma$;

Vмез - удельный объем мезопор, $\mathrm{cm}^{3} / \Gamma$;

Vмак - удельный объем макропор, $\mathrm{cm}^{3} / \Gamma$;

Vпор - общий объем пор, $\mathrm{cm}^{3} /$ г.

Условия и результаты эксперимента приведены в табл. 1.

\section{Результаты и обсуждение}

В табл. 1 приведены результаты полученных экспериментальных величин.

Результаты математической обработки для каждого выходного параметра в отдельности приведены ниже. В табл. 2 даны результаты дисперсионного анализа для $\mathrm{S}_{\text {Бэт. }}$

Таблица 1. Влияние состава исходной смеси компонентов на характеристики получаемых органических лигнин-фенол-формальдегидных аэрогелей

\begin{tabular}{|c|c|c|c|c|c|}
\hline \multicolumn{2}{|c|}{$\begin{array}{c}\text { Массовое соотношение } \\
\text { компонентов }\end{array}$} & \multicolumn{4}{|c|}{ Величины выходных параметров процесса } \\
\hline $\begin{array}{c}\Phi е н / Л \\
\mathrm{X}_{1}\end{array}$ & $\begin{array}{c}(\text { Л+Фен }) / \Phi \\
\mathrm{X}_{2}\end{array}$ & $\begin{array}{c}\text { удельная } \\
\text { поверхность, } \\
\mathrm{S}_{\text {гэт, }} \mathrm{M}^{2} / \Gamma \\
\end{array}$ & $\begin{array}{l}\text { объем мезопор, } \\
\text { Умез, } \mathrm{cm}^{3} / \Gamma\end{array}$ & $\begin{array}{c}\text { объем } \\
\text { макропор, } \\
\text { Vмак, } \text { см }^{3} / \Gamma \\
\end{array}$ & 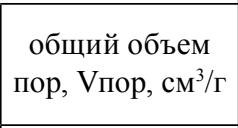 \\
\hline 0,25 & 1,7 & 334 & 1,29 & 2,24 & 3,63 \\
\hline 0,43 & 1,7 & 400 & 2,26 & 0,46 & 2,86 \\
\hline 0,67 & 1,7 & 451 & 1,58 & 0,51 & 2,23 \\
\hline 1,0 & 1,7 & 486 & 1,18 & 0,39 & 1,72 \\
\hline 1,5 & 1,7 & 176 & 0,10 & 0,25 & 0,41 \\
\hline 0,43 & 1,25 & 269 & 0,82 & 3,14 & 4,05 \\
\hline 0,67 & 1,25 & 371 & 1,56 & 1,61 & 3,29 \\
\hline 1,0 & 1,25 & 405 & 1,81 & 0,79 & 2,73 \\
\hline 1,5 & 1,25 & 198 & 0,21 & 0,17 & 0,44 \\
\hline
\end{tabular}




\section{Удельная поверхность}

Дисперсионный анализ выявил, что существенный вклад в суммарную дисперсию выходного параметра вносят оба фактора. На это указывают высокие значения дисперсионных отношений (они определяются как отношение средних квадратов, связанных с каждым из источников дисперсии, к остаточной сумме средних квадратов), называемые также эффективностями влияния. Считается (хотя это не формализовано), что в большинстве случаев приемлемой можно считать эффективность $F>5 \ldots 8$. Аналогично интерпретируется информация, содержащаяся в последнем столбце таблицы. Влияние источника дисперсии на выходной параметр считается статистически значимым, если уровень значимости меньше 0,05 ; этому соответствует доверительная вероятность $(1-0,05) \cdot 100=95 \%$. В рассматриваемой задаче только значимость вклада от источника дисперсии $\mathrm{X}_{1}$ оценивается с меньшей доверительной вероятностью, равной $(1-0,1459) \cdot 100=85 \%$.

Зависимость $\mathrm{S}_{\text {Бэт }}$ от переменных факторов процесса аппроксимирована уравнением регрессии второго порядка

$$
S_{\text {Бэт }}=-653+1486 X_{1}+457 X_{2}-590 X_{1}^{2}-330 X_{1} X_{2} \text {. }
$$

Качество аппроксимации характеризуется коэффициентом детерминации $\mathrm{R}^{2}$ (близким по смыслу к коэффициенту множественной корреляции между выходным параметром $\mathrm{S}_{\text {Бэт и не- }}$ зависимыми переменными). В рассматриваемой задаче этот коэффициент имеет высокое значение $\mathrm{R}^{2}=97,9 \%$, что может указывать на хорошее качество аппроксимации (максимально возможная величина коэффициента детерминации 100 \%). Следует, однако, иметь в виду, что величина этого показателя зависит от числа степеней свободы: при большом числе степеней свободы эта зависимость проявляется слабо и не влияет в заметной степени на интерпретацию результатов, однако при небольшом числе опытов величина $\mathrm{R}^{2}$ может оказаться очень завышенной. В этом случае более надежной будет оценка $\mathrm{R}_{\mathrm{adj}}{ }^{2} \mathrm{c}$ поправкой на число степеней свободы (последняя строка в таблице дисперсионного анализа). В рассматриваемом случае величины $\mathrm{R}^{2}$ и $\mathrm{R}_{\mathrm{adj}}{ }^{2}$ достаточно близки между собой и к $100 \%$. Это позволяет говорить об адекватности

Таблица 2. Сводка дисперсионного анализа для удельной поверхности органических аэрогелей из лигнина

\begin{tabular}{|c|c|c|c|c|c|}
\hline $\begin{array}{l}\text { Источники } \\
\text { дисперсии }\end{array}$ & $\begin{array}{c}\text { Суммы } \\
\text { квадратов }\end{array}$ & $\begin{array}{l}\text { Степени } \\
\text { свободы }\end{array}$ & $\begin{array}{l}\text { Средние } \\
\text { квадраты }\end{array}$ & $\begin{array}{c}\text { Дисперсионные } \\
\text { отношения } \boldsymbol{F}\end{array}$ & $\begin{array}{c}\text { Уровни } \\
\text { значимости } \boldsymbol{P}\end{array}$ \\
\hline $\mathrm{X}_{1}:$ Фен/Л & 1616,1 & 1 & 1616,1 & 3,25 & 0,1459 \\
\hline $\mathrm{X}_{2}:(Л+Ф е н) / \Phi$ & 11912,0 & 1 & 11912,0 & 23,93 & 0,0081 \\
\hline $\mathrm{X}_{1}^{2}$ & 67192,3 & 1 & 67192,3 & 134,99 & 0,0003 \\
\hline $\mathrm{X}_{1} \mathrm{X}_{2}$ & 8007,6 & 1 & 8007,7 & 16,09 & 0,0160 \\
\hline Остаточная сумма & 1991,0 & 4 & 497,7 & - & - \\
\hline Общая сумма & 94460,0 & 8 & - & - & - \\
\hline $\mathrm{R}^{2}, \%$ & 97,9 & - & - & - & - \\
\hline $\begin{array}{l}\mathrm{R}_{\mathrm{adj}}{ }^{2} \text { с поправкой на число } \\
\text { степеней свободы, \% }\end{array}$ & 95,8 & - & - & - & - \\
\hline
\end{tabular}


уравнения (1) результатам наблюдений и использовать его в качестве математической модели изучаемого процесса.

Прогностические свойства уравнения (1) наглядно демонстрируются рис. 1, на котором сравниваются значения выходного параметра $\mathrm{S}_{\text {Бэт }}$, наблюдавшиеся в эксперименте, со значениями, прогнозируемыми по модели.

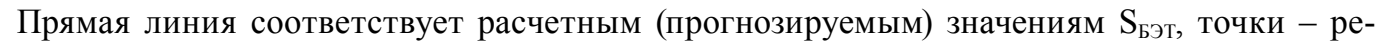
зультатам наблюдений. Близость «экспериментальных точек» к прямой линии подтверждает хорошие прогностические свойства уравнения (1).

Математическая модель использована для графического отображения (в виде поверхности отклика) зависимости выходного параметра $\mathrm{S}_{\text {Бэт }}$ от переменных факторов $\mathrm{X}_{1}$ и $\mathrm{X}_{2}$ (рис. 2).

На рис. 2 приведены двумерные сечения трехмерной поверхности отклика, наглядно демонстрирующие влияние данных переменных факторов на выходной параметр $\mathrm{S}$ Бэт. Точка «оптимума» соответствует максимальному прогнозируемому значению в изученной области факторного пространства. Максимальное прогнозируемое значение $\mathrm{S}_{\text {Бэт }}=485 \mathrm{~m}^{2} / \Gamma$ достигается

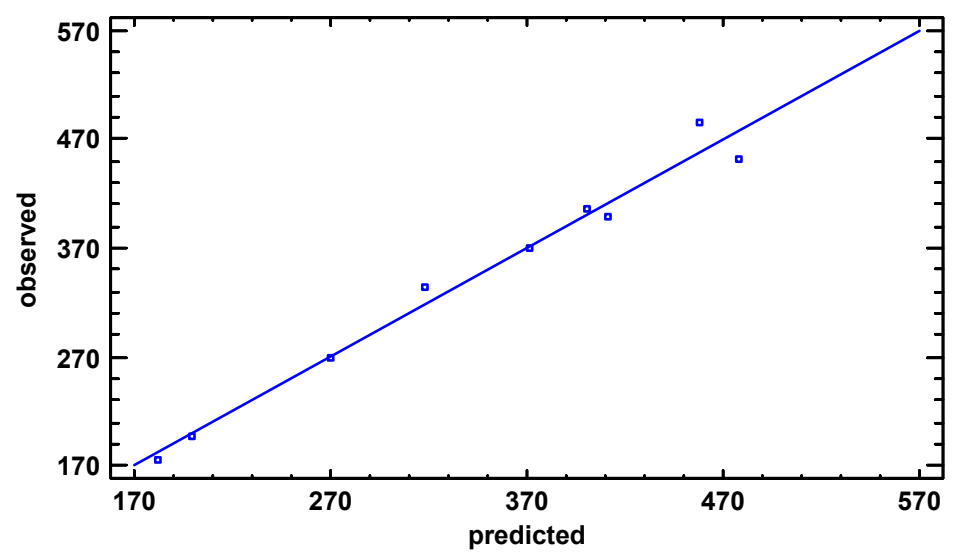

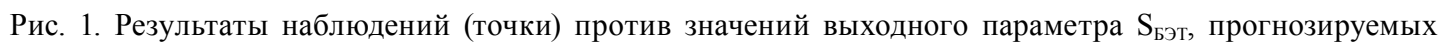
математической моделью

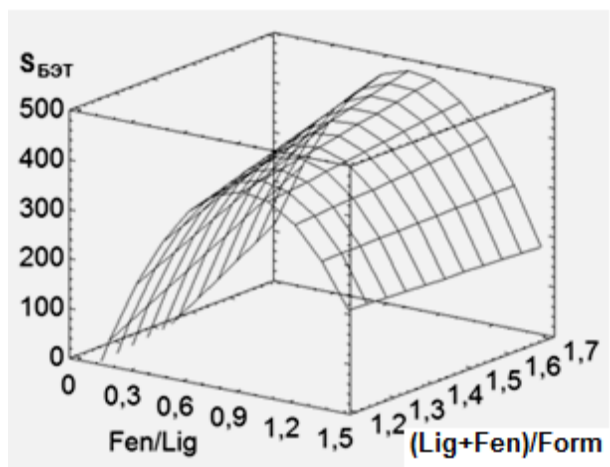

Рис. 2. Поверхность отклика зависимости $\mathrm{S}_{\text {Бэт }}$ от переменных факторов процесса 
согласно вычислениям по математической модели в точке, соответствующей следующим значениям переменных факторов: соотношение Фен/Л (фактор $\mathrm{X}_{1}$ ) 0,783 ; соотношение (Л + Фен)/Ф (фактор $\left.\mathrm{X}_{2}\right) 1,70$.

\section{Объем мезопор}

В табл. 3 приведены результаты дисперсионного анализа для объема мезопор органических аэрогелей из лигнина.

Результаты дисперсионного анализа указывают на сравнительно слабое влияние переменных факторов на обсуждаемый выходной параметр (невысокий коэффициент детерминации, низкие эффективности источников дисперсии). Уравнение регрессии, аппроксимирующее зависимость Vмез от $\mathrm{X}_{1}$ и $\mathrm{X}_{2}$, имеет вид

$$
\text { Vмез }=-3,596+7,670 X_{1}+2,548 X_{2}-2,685 X_{1}^{2}-2,476 X_{1} X_{2} .
$$

Рисунок 3 демонстрирует удовлетворительное соответствие расчетных значений результатам эксперимента, однако «разброс» точек вокруг прямой заметно больше, чем в случае анализа $\mathrm{S}_{\text {вэт }}$.

Характер отклонений точек (равномерное распределение вдоль прямой) позволяет предполагать, что основной причиной сравнительно низкой эффективности модели является экспериментальный «шум». Влияние шума может быть вызвано остаточным присутствием молекул жидкости, находящихся в порах аэрогеля, при их замене на чистый этанол либо сдавливании гелеобразной массы при нарезании образцов в виде блоков.

На основании математического моделирования было определено, что наиболее существенные линейные корреляции существуют между удельной поверхностью образцов $\mathrm{S}_{\text {Бэт }}$ и мезопористым объемом Vмез. Как следствие, условия, при которых достигаются максималь-

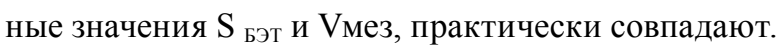

Поверхность отклика зависимости выходного параметра Vмез от переменных факторов $\mathrm{X}_{1}$ и $\mathrm{X}_{2}$ изображена на рис. 4. Можно отметить внешнее сходство поверхностей, изображенных

Таблица 3. Сводка дисперсионного анализа для объема мезопор органических аэрогелей

\begin{tabular}{|c|c|c|c|c|c|}
\hline $\begin{array}{c}\text { Источники } \\
\text { дисперсии }\end{array}$ & $\begin{array}{c}\text { Суммы } \\
\text { квадратов }\end{array}$ & $\begin{array}{l}\text { Степени } \\
\text { свободы }\end{array}$ & $\begin{array}{c}\text { Средние } \\
\text { квадраты }\end{array}$ & $\begin{array}{c}\text { Диспер- } \\
\text { сионные } \\
\text { отношения } F\end{array}$ & $\begin{array}{c}\text { Уровни } \\
\text { значимости } P\end{array}$ \\
\hline $\mathrm{X}_{1}:$ Фен/Л & 0,655 & 1 & 0,655 & 3,49 & 0,1352 \\
\hline $\mathrm{X}_{2}:(Л+Ф е н) / \Phi$ & 0,062 & 1 & 0,062 & 0,33 & 0,5963 \\
\hline $\mathrm{X}_{1}^{2}$ & 1,391 & 1 & 1,391 & 7,40 & 0,0529 \\
\hline $\mathrm{X}_{1} \mathrm{X}_{2}$ & 0,449 & 1 & 0,449 & 2,39 & 0,1971 \\
\hline Остаточная сумма & 0,752 & 4 & 0,188 & - & - \\
\hline Общая сумма & 4,113 & 8 & - & - & - \\
\hline $\mathrm{R}^{2}, \%$ & 81,7 & - & - & - & - \\
\hline $\begin{array}{l}\mathrm{R}_{\mathrm{adj}}^{2} \text { с поправкой } \\
\text { на число степеней } \\
\text { свободы, \% }\end{array}$ & 63,4 & - & - & - & - \\
\hline
\end{tabular}




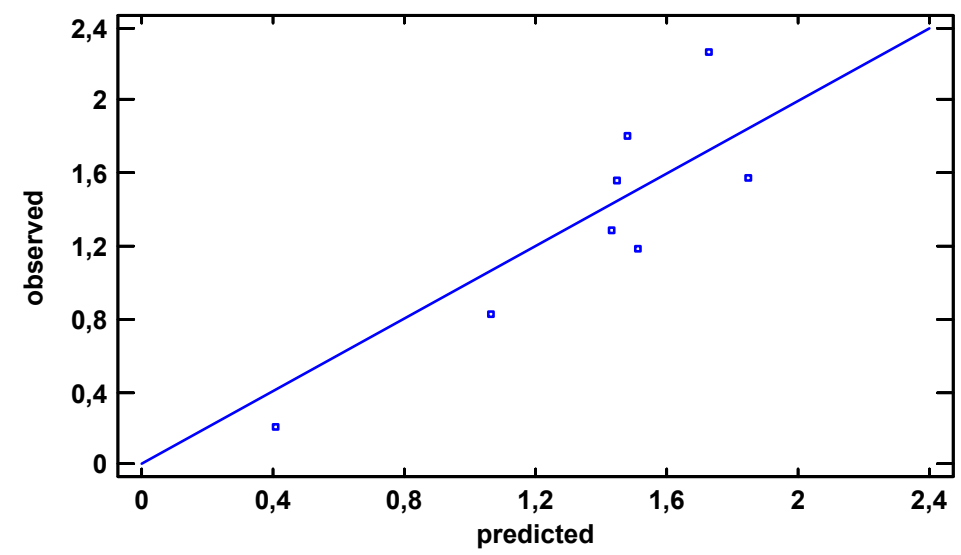

Рис. 3. Результаты наблюдений (точки) против значений выходного параметра Vмез, прогнозируемых математической моделью

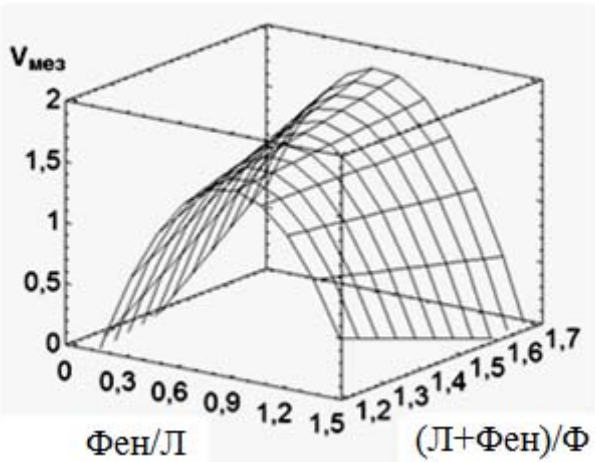

Рис. 4. Поверхность отклика зависимости Vмез от переменных факторов процесса

на рис. 2 и 4, что может указывать на одинаковую физическую природу характеристик $\mathrm{S}_{\text {Бэт }}$ и Vмез.

Максимальное прогнозируемое значение $\mathrm{Vme} 3=1,85 \mathrm{~cm}^{3} / \Gamma$ в изученной области факторного пространства достигается согласно вычислениям по математической модели в точке, соответствующей следующим значениям переменных факторов: соотношение Fen/Lig (фактор $\left.\mathrm{X}_{1}\right)$ 0,645; соотношение $(\mathrm{Lig}+\mathrm{Fen}) /$ Form (фактор $\left.\mathrm{X}_{2}\right)$ 1,700. Как и следовало ожидать, учитывая изложенное выше обстоятельство, наибольшие значения удельной поверхности и объема мезопор достигаются при почти одинаковых условиях получения аэрогеля.

\section{Объем макропор}

В табл. 4 приведены результаты дисперсионного анализа объема макропор органических аэрогелей из лигнина.

Дисперсионный анализ показал, что существенный вклад в суммарную дисперсию выходного параметра вносят все источники дисперсии. Уравнение регрессии, аппроксимирующее зависимость Vмакро от $\mathrm{X}_{1}$ и $\mathrm{X}_{2}$, имеет вид 
Таблица. 4. Сводка дисперсионного анализа объема макропор органических аэрогелей

\begin{tabular}{|c|c|c|c|c|c|}
\hline $\begin{array}{l}\text { Источники } \\
\text { дисперсии }\end{array}$ & $\begin{array}{c}\text { Суммы } \\
\text { квадратов }\end{array}$ & $\begin{array}{l}\text { Степени } \\
\text { свободы }\end{array}$ & $\begin{array}{c}\text { Средние } \\
\text { квадраты }\end{array}$ & $\begin{array}{c}\text { Диспер- } \\
\text { сионные } \\
\text { отношения } \\
F\end{array}$ & $\begin{array}{c}\text { Уровни } \\
\text { значимости } \\
P\end{array}$ \\
\hline $\mathrm{X}_{1}:$ Фен/Л & 6,479 & 1 & 6,479 & 29,96 & 0,0054 \\
\hline $\mathrm{X}_{2}:(Л+\Phi е н) / \Phi$ & 1,997 & 1 & 1,997 & 9,23 & 0,0385 \\
\hline $\mathrm{X}_{1}^{2}$ & 1,289 & 1 & 1,289 & 5,96 & 0,0711 \\
\hline $\mathrm{X}_{1} \mathrm{X}_{2}$ & 1,395 & 1 & 1,395 & 6,45 & 0,0640 \\
\hline Остаточная сумма & 0,865 & 4 & 0,216 & - & - \\
\hline Общая сумма & 8,653 & 8 & - & - & - \\
\hline $\mathrm{R}^{2}, \%$ & 90,0 & - & - & - & - \\
\hline $\begin{array}{l}\mathrm{R}_{\mathrm{adj}}^{2} \text { с поправкой на число } \\
\text { степеней свободы, \% }\end{array}$ & 80,0 & - & - & - & - \\
\hline
\end{tabular}

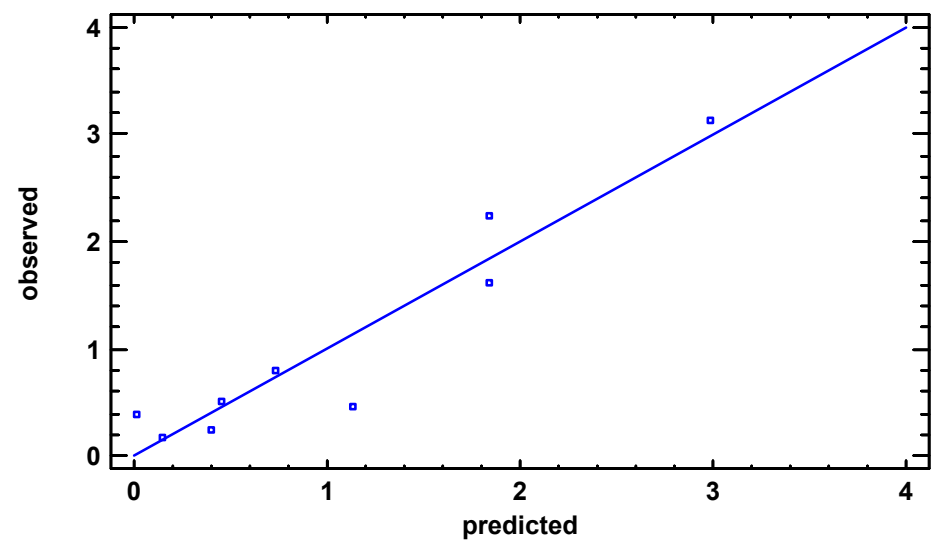

Рис. 5. Результаты наблюдений (точки) против значений выходного параметра Vмакро, прогнозируемых математической моделью

$$
\text { Vмакро }=13,280-13,098 \mathrm{X}_{1}-5,986 \mathrm{X}_{2}+2,584 \mathrm{X}_{2}^{2}+4,364 \mathrm{X}_{1} \mathrm{X}_{2} \text {. }
$$

Высокое значение коэффициента детерминации (табл. 4) и близость большинства точек к прямой (рис. 5) указывают на хорошие прогностические свойства математической модели.

Поверхность отклика зависимости выходного параметра Умакро от переменных факторов $\mathrm{X}_{1}$ и $\mathrm{X}_{2}$ изображена на рис. 6 . Вид поверхности отклика значительно отличается от зависимостей, приведенных на рис. 2 и 4.

Максимальное прогнозируемое значение $\mathrm{VMa \kappa}=4,05 \mathrm{~cm}^{3} / \Gamma$ в изученной области факторного пространства достигается согласно вычислениям по математической модели в точке, соответствующей следующим значениям переменных факторов: соотношение Фен/Л (фактор $\mathrm{X}_{1}$ ) 0,25 ; соотношение (Л + Фен)/Ф (фактор $\left.\mathrm{X}_{2}\right)$ 1,25. Эти условия существенно отличаются от условий, при которых достигаются максимальные значения $\mathrm{S}_{\text {Бэт }}$ и $\mathrm{Vмез.}$

$$
-295-
$$




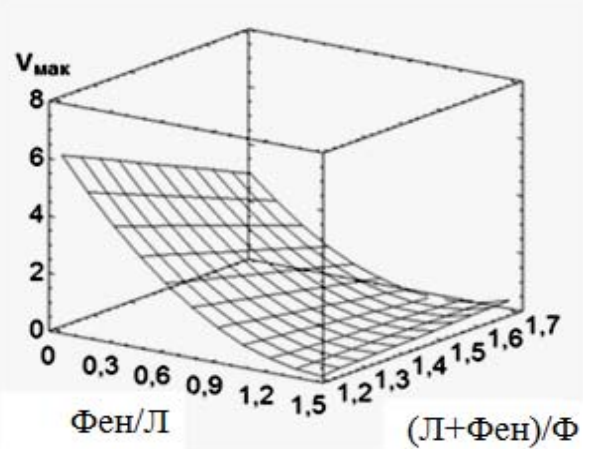

Рис. 6. Поверхность отклика зависимости Vмакро от переменных факторов процесса

\section{Общий объем пор}

Результаты дисперсионного анализа общего объема пор органических аэрогелей из лигнина приведены в табл. 5 .

Дисперсионный анализ показал, что существенный вклад в суммарную дисперсию выходного параметра вносят только главные эффекты. Вклад парного взаимодействия факторов $\mathrm{X}_{1} \mathrm{X}_{2}$ сравнительно слабый (эффективность $F=2,77$ ), а $\mathrm{X}_{1}^{2}$ практически не вносит вклада в суммарную дисперсию выходного параметра (его эффективность $F=0,18$ ), т. е. не оказывает влияния на Vпор.

Высокое значение коэффициента детерминации (табл. 5) и близость точек к прямой (рис. 7) указывают на хорошие прогностические свойства математической модели.

Уравнение регрессии, аппроксимирующее зависимость Vпор от $\mathrm{X}_{1}$ и $\mathrm{X}_{2}$, имеет вид

$$
\text { Vпор }=9,510-4,994 \mathrm{X}_{1-} 3,311 \mathrm{X}_{2}-0,284 \mathrm{X}_{1}^{2}+1,802 \mathrm{X}_{1} \mathrm{X}_{2} \text {. }
$$

Поверхность отклика зависимости выходного параметра Упор от переменных факторов $\mathrm{X}_{1}$ и $\mathrm{X}_{2}$ изображена на рис. 8.

Таблица 5. Сводка дисперсионного анализа общего объема пор органических аэрогелей

\begin{tabular}{|c|c|c|c|c|c|}
\hline $\begin{array}{l}\text { Источники } \\
\text { дисперсии }\end{array}$ & $\begin{array}{c}\text { Суммы } \\
\text { квадратов }\end{array}$ & $\begin{array}{l}\text { Степени } \\
\text { свободы }\end{array}$ & $\begin{array}{l}\text { Средние } \\
\text { квадраты }\end{array}$ & $\begin{array}{c}\text { Диспер- } \\
\text { сионные } \\
\text { отношения } F\end{array}$ & $\begin{array}{c}\text { Уровни } \\
\text { значимости } \\
P\end{array}$ \\
\hline $\mathrm{X}_{1}:$ Fen/Lig & 11,3678 & 1 & 11,3678 & 132,57 & 0,0003 \\
\hline $\mathrm{X}_{2}:($ Lig+Fen $) /$ Form & 1,27827 & 1 & 1,27827 & 14,91 & 0,0181 \\
\hline $\mathrm{X}_{1}^{2}$ & 0,0155919 & 1 & 0,0155919 & 0,18 & 0,6918 \\
\hline $\mathrm{X}_{1} \mathrm{X}_{2}$ & 0,237862 & 1 & 0,237862 & 2,77 & 0,1711 \\
\hline Остаточная сумма & 0,343002 & 4 & 0,0857504 & - & - \\
\hline Общая сумма & 13,6346 & 8 & - & - & - \\
\hline $\mathrm{R}^{2}, \%$ & \multicolumn{5}{|c|}{97,5} \\
\hline $\begin{array}{l}\mathrm{R}_{\text {adj }}{ }^{2} \text { с поправкой на число } \\
\text { степеней свободы, } \%\end{array}$ & \multicolumn{5}{|c|}{95,0} \\
\hline
\end{tabular}




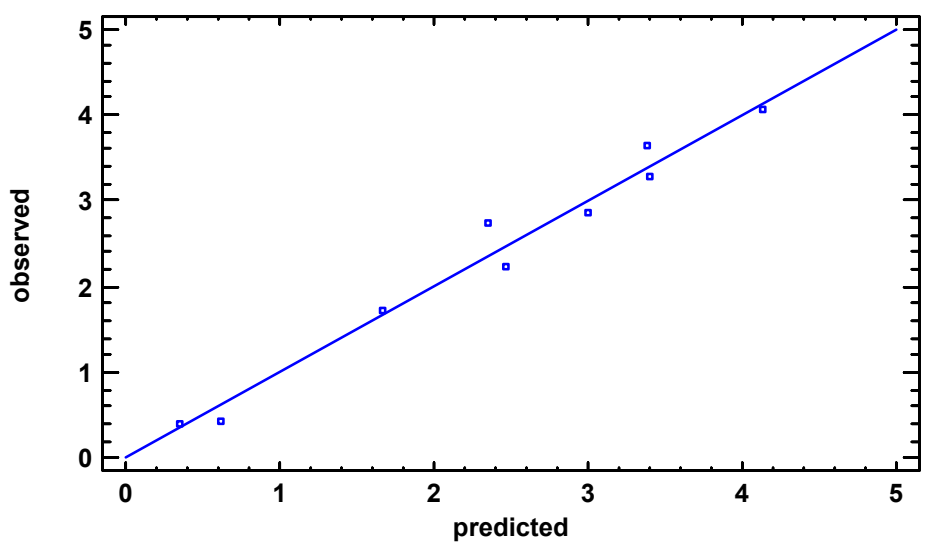

Рис. 7. Результаты наблюдений (точки) против значений выходного параметра Vпор, прогнозируемых математической моделью

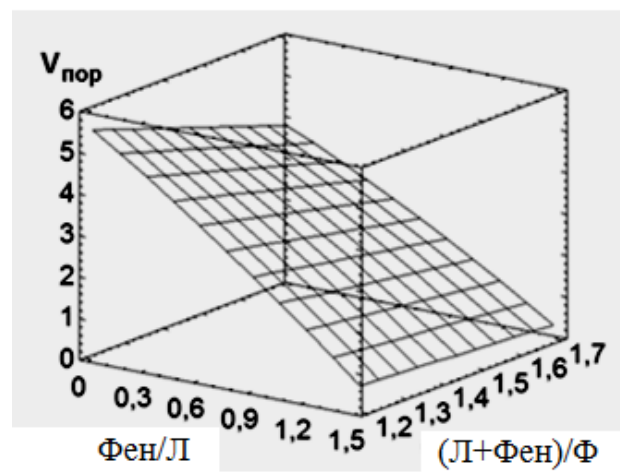

Рис. 8. Поверхность отклика зависимости Vпор от переменных факторов процесса

По указанной выше причине (незначительный вклад нелинейных эффектов) она похожа на плоскость с наклоном в ту же сторону, что поверхность отклика на рис. 6. Очевидно, обе характеристики, Умак и Упор, имеют одну и ту же природу.

Максимальное прогнозируемое значение $V$ пор $=4,67 \mathrm{~cm}^{3} / \Gamma$ в изученной области факторного пространства достигается согласно вычислениям по математической модели в точке, соответствующей следующим значениям переменных факторов: соотношение Фен/Л (фактор $\mathrm{X}_{1}$ ) 0,25; соотношение (Л + Фен)/Ф (фактор $\mathrm{X}_{2}$ ) 1,25. Эти условия, естественно, близки к условиям, при которых достигается максимум Vмак, и существенно отличаются от условий, при которых достигаются максимальные значения $\mathrm{S}_{\text {Бэт }}$ и Vмез.

\section{Вычисление оптимальных условий}

Визуальное сравнение связей между выходными параметрами подтверждается вычислением коэффициентов парных линейных корреляций (табл. 6).

Наиболее существенные линейные корреляции существуют между парой $\mathrm{S}_{\text {Бэт }}$ и $\mathrm{V}_{\text {мез, }}$ a также парой Vмак и Vпор, тогда как между собой эти две пары связаны слабо или связь

$$
-297-
$$


Таблица 6. Матрица коэффициентов корреляций между выходными параметрами

\begin{tabular}{|c|c|c|c|c|}
\hline & $\mathrm{S}_{\text {Бэт }}$ & Умез & Умак & Vпор \\
\hline $\mathrm{S}_{\text {Бэт }}$ & 1 & 0,804 & $-0,101$ & 0,387 \\
\hline Умез & 0,804 & 1 & 0,797 & 0,303 \\
\hline Умак & $-0,101$ & 0,797 & 1 & 0,821 \\
\hline Упор & 0,387 & 0,303 & 0,821 & 1 \\
\hline
\end{tabular}

вообще отсутствует. Следствие этого: условия, при которых достигаются максимальные

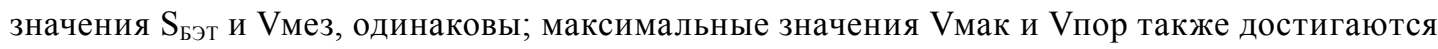
при одинаковых условиях; те и другие условия не совпадают, о чем уже упоминалось. Следовательно, за оптимальный режим можно принять любой из двух режимов, в зависимости от того, какие из свойств продукта наиболее ценны. Если при оптимизации процесса необходимо одновременно учитывать несколько изложенных выше свойств аэрогеля, возникает компромиссная задача, условия которой формулируются в терминах математического программирования.

В рассматриваемом случае был сформулирован следующий критерий оптимальности

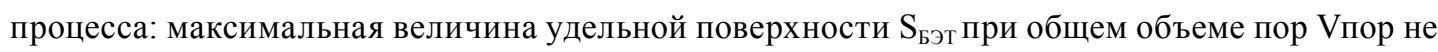
менее $1,7 \mathrm{~cm}^{3} /$ г и удельном объеме мезопор Vмез не менее $1,2 \mathrm{~cm}^{3} /$ г.

Математическая формулировка задачи нелинейного программирования: целевая функция

$\mathrm{S}_{\text {Бэт }} \rightarrow \max ;$

ограничения на другие выходные параметры

Упор $\geq 1,7$

Умез $\geq 1,2 ;-$

ограничение области поиска

$0,25 \leq$ Фен/Л $\leq 1,5 ;$

$1,25 \leq(Л+Ф е н) / \Phi \leq 1,70$.

Решение задачи, полученное градиентным методом [20]:

Фен/Л = 1,0;

$($ Л+Фен $) / \Phi=1,7$.

Это решение совпадает с условиями одного из опытов (см. табл. 1, 4-я строка).

\section{Заключение}

В результате экспериментально-статистического анализа получены математические модели, описывающие влияние независимых факторов процесса (массового соотношения исходных реагентов - фенола и лигнина - и массового соотношения смеси лигнина с фенолом и формальдегида) на значения конкретных выходных параметров пористой структуры получаемых органических лигнин-фенол-формальдегидных аэрогелей, характеризующих такие свойства продукта, как удельная поверхность, пористый объем, объем мезо- и макропор. 
На основании оценки полученной математической модели в пределах изученного факторного пространства установлено, что наиболее существенные линейные корреляции существуют между удельной поверхностью $\mathrm{S}_{\text {Бэт }}$ органических лигнин-фенол-формальдегидных аэрогелей и объемом мезопор Vмез. Установлено, что выходные параметры процесса $\mathrm{S}_{\text {Бэт и } \mathrm{Vмез}}$ достигают своих наилучших значений практически в одинаковых оптимальных условиях получения органического аэрогеля.

Прогнозируемый результат процесса при следующих условиях:

- $\quad$ (фактор $\left.\mathrm{X}_{1}\right)$ соотношение фенол/лигнин Фен/Л 0,65 ... 0,78 (по массе);

- $\quad\left(\right.$ факторХ $\left.\mathrm{X}_{2}\right)$ соотношение лигнин+фенол/формальдегид $(Л+\Phi е н) / \Phi=1,7$ выглядят следующим образом:

- прогнозируемая величина удельной поверхности $\mathrm{S}_{\text {Бэт }} 485 \mathrm{~m}^{2} / \Gamma$;

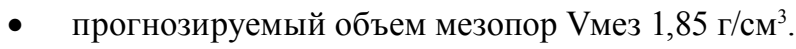

В соответствии с полученной математической моделью установлено, что максимальные прогнозируемые значения Vмак и Упор также достигаются при одинаковых условиях, в точке оптимума, соответствующей следующим значениям переменных факторов:

- с соотношение фенол/лигнин Фен/Л (фактор $\mathrm{X}_{1}$ ) 0,25;

- соотношение лигнин+ фенол/формальдегид (Л+Фен)/Ф (фактор $\left.\mathrm{X}_{2}\right)=1,25$.

Рассчитаны максимальные прогнозируемые значения выходных параметров:

- $\mathrm{Vмак}=4,05 \mathrm{~cm}^{3} / \Gamma$;

- Ипор $=4,67 \mathrm{~cm}^{3} / \Gamma$.

Следовательно, на основании математического моделирования за оптимальный режим можно принять любой из двух режимов, влияющих на лучшие параметры пористости аэрогелей в зависимости от того, какие конкретные свойства продукта наиболее ценны. Установлено, что прогнозируемые условия получения органических лигнинфенол-формальдегидных аэрогелей сопоставимы с экспериментально полученными значениями.

Таким образом, предсказанные уравнениями регрессии значения выходных параметров, характеризующих пористую структуру получаемых органических лигнин-фенолформальдегидных аэрогелей, отражают адекватность полученных математических моделей, связывающих значения выходных факторов и результаты эксперимента, и целесообразность их использования для прогнозирования результатов процесса.

Изучением подбора оптимальных условий синтеза аэрогелей из доступных и экологически безопасных возобновляемых источников природного органического сырья можно расширить сферу их потенциального применения.

\section{Список литературы}

1. Wu D., Fu R., Sun Z., Yu Z. Low-density organic and carbon aerogels from the sol-gel polymerization of phenol with formaldehyde // Journal of Non-Crystalline Solids. 2005. Vol. 351. № 10-11. P. 915-921.

2. García-González C.A., Alnaief M., Smirnova I. Polysaccharide-based aerogels-Promising biodegradable carriers for drug delivery systems // Carbohydrate Polymers. 2011. Vol. 86. № 4. P. $1425-1438$. 
3. Amaral-Labat G., Grishechko L.I., Szczurek A., et al. Highly mesoporous organic aerogels derived from soy and tannin // Green Chemistry. 2012. Vol.14. P. 3099-106.

4. Escudero R.R., Robitzer M., Di Renzo F., et al. Alginate aerogels as adsorbents of polar molecules from liquid hydrocarbons: Hexanol as probe molecule // Carbohydrate Polymers. 2009. Vol. 75. P. 52-57.

5. Peirano F., Vincent T., Quignard F., et al. Palladium supported on chitosan hollow fiber for nitrotoluene hydrogenation // Journal of Membrane Science. 2009. Vol. 239. № 1-2. P. 30-45.

6. Al-Muhtaseb S.A., Ritter J.A. Preparation and Properties of Resorcinol-Formaldehyde Organic and Carbon Gels // Advanced Materials. 2003. Vol. 15. № 2. P. 101-114.

7. Job N., Panariello F., Marien C.M., et al. Synthesis optimization of organic xerogels produced from convective air-drying of resorcinol-formaldehyde gels // Journal of Non-Crystalline Solids. 2006. Vol. 325. P. 24-34.

8. Gaca K.Z., Sefcik J. Mechanism and kinetics of nanostructure evolution during early stages of resorcinol-formaldehyde polymerization // Journal of Colloid and Interface Science. 2013. Vol. 406. P. 51-59.

9. Fischer F., Rigacci A., Pirard R., et al. Cellulose-based aerogels // Polymer. 2006. Vol. 47. № 22. P. 7636-7645.

10. Aaltonen O., Jauhiainen O. The preparation of lignocellulosic aerogels from ionic liquid solutions // Carbohydrate Polymers. 2009. Vol. 75. № 1. P. 125-129.

11. Son T. Nguyen, Jingduo Feng, Shao Kai Ng, et al. Advanced thermal insulation and absorption properties of recycled cellulose aerogels // Colloids and Surfaces A: Physicochemical and Engineering Aspects. 2014. Vol. 445. P. 128-134.

12. Tsioptsias C., Michailof C., Stauropoulos G., Panayiotou C. Chitin and carbon aerogels from chitin alcogels // Carbohydrate Polymers. 2009. Vol.76. № 4. P. 535-540.

13. Comin L.M., Temelli F., Saldaña M.D.A. Barley beta-glucan aerogels via supercritical CO2 drying // Food Research International. 2012. Vol. 48. №.2. P. 442-448.

14. Scherdel C., Reichenauer G. Carbon xerogels synthesized via phenol-formaldehyde gels // Microporous and Mesoporous Materials. 2009. Vol. 126. P.133-142.

15. Martín C.F., Plaza M.G., García S., et al. Microporous phenol-formaldehyde resin-based adsorbents for pre-combustion CO2 capture // Fuel. 2011. Vol. 90. № 5. P. 2064-2072.

16. Ping L., Pizzi A., Guo Z., Brosse N. Condensed tannins extraction from grape pomace: Characterization and utilization as wood adhesives for wood particleboard // Industrial Crops and Products. 2011. Vol. 34. № 1. P. 907-914.

17. Pizzi A. Tannins: Major Sources, Properties and Applications // In: Monomers, Polymers and Composites from renewable resources / Ed. by M.N. Belgacem, A. Gandini. Elsevier. 2008. P. 179-199.

18. G. Amaral-Labat, L.I. Grishechko, V. Fierro, B.N. Kuznetsov, A. Pizzi, A. Celzard. Tanninbased xerogels with distinctive porous structures// Biomass and Bioenergy. 2013. Vol. 56. P. 437-445.

19. Пен Р.3. Планирование эксперимента в Statgraphics Centurion. Красноярск, 2014. 293 с. / Pen R.Z. Experimental Design in Statgraphics Centurion. Krasnoyarsk, 2014. 293 p.

20. Курицкий Б.Я. Поиск оптимальных решений средствами Excel в примерах. СПб., 1997. 384 c. / Kuritskij B.Ya. The search for optimal solutions by means of Excel in the examples. St. Petersburg, 1997. 384 p. 